\title{
Universiteit
}

Leiden

The Netherlands

\section{Global analysis of trait-trait relationships within and between species}

Zhou, J.; Cieraad, E.; Bodegom, P.M. van

\section{Citation}

Zhou, J., Cieraad, E., \& Bodegom, P. M. van. (2021). Global analysis of trait-trait

relationships within and between species. New Phytologist. doi:10.1111/nph.17879

Version: $\quad$ Publisher's Version

License: $\quad$ Creative Commons CC BY-NC 4.0 license

Downloaded from: https://hdl.handle.net/1887/3249255

Note: To cite this publication please use the final published version (if applicable). 


\title{
Global analysis of trait-trait relationships within and between
} species

\author{
Jianhong Zhou ${ }^{1}$ (D), Ellen Cieraad ${ }^{1,2}$ (iD) and Peter M. van Bodegom ${ }^{1}$ \\ ${ }^{1}$ Institute of Environmental Sciences (CML), Leiden University, 2333 CC Leiden, the Netherlands; ${ }^{2}$ Nelson Marlborough Institute of Technology, 322 Hardy Street, Nelson 7010 , \\ New Zealand
}

Author for correspondence:

Jianhong Zhou

Email: j.zhou@cml.leidenuniv.nl

Received: 28 July 2021

Accepted: 16 November 2021

New Phytologist (2021)

doi: 10.1111/nph.17879

Key words: common environmental drivers, global meta-analysis, interspecific trait variation, intraspecific trait variation, plant strategies, trait-trait relationships.

\section{Summary}

- Some commonly reported trait-trait relationships between species, including the leaf economic spectrum (LES), are regarded as important plant strategies but whether these relationships represent plant strategies in reality remains unclear.

- We propose a novel approach to distinguish trait-trait relationships between species that may represent plant strategies vs those relationships that are the result of common drivers, by comparing the direction and strength of intraspecific trait variation (ITV) vs interspecific trait variation. We applied this framework using a unique global ITV database that we compiled, which included 11 traits related to LES, size and roots, and observations from 2064 species occurring in 1068 communities across 19 countries.

- Generally, compared to between species, trait-trait relationships within species were much weaker or totally disappeared. Almost only within the LES traits, the between-species traittrait relationships were translated into positive relationships within species, which suggests that they may represent plant strategies.

- Moreover, the frequent coincidental trait-trait relationships between species, driven by covarying common drivers, imply that in future research, decoupling of trait-trait relationships should be considered seriously in model projections of ecosystem functioning. Our study emphasizes the importance of describing the mechanisms behind trait-trait relationships, both between and within species, for deepening our understanding of general plant strategies.

\section{Introduction}

Despite widely recognized trait-trait relationships between plant species, including plant trait correlations that define the so-called global leaf economics spectrum (LES), it remains unclear whether these economics spectra represent true coordination or trade-offs (Grubb, 2016). Leaf economics spectrum describes the multivariate relationships between six leaf traits (leaf mass per area (LMA), photosynthetic assimilation rates $\left(A_{\text {mass }}\right.$ or $\left.A_{\text {area }}\right)$, leaf nitrogen $(\mathrm{N})$, leaf phosphorus $(\mathrm{P})$, dark respiration rate $\left(R_{\text {mass }}\right.$ or $R_{\text {area }}$ and leaf lifespan (LL)) and runs from a fast to a slow return of plant leaf investment among species (Wright $e t$ al., 2004; Westoby et al., 2013). Leaf economics spectrum has been suggested to represent plant strategies, and it has been variably extended to include other plant organs such as stem economics spectrum (SES), root economics spectrum (RES), and even to the whole plant economics spectrum (PES) (Freschet et al., 2010; Reich, 2014). If trait relationships arise through physiological mechanisms or eco-evolutionary constraints and, thus, represent plant strategies, these trait relationships can help, for example, predict how plants respond to projected changes in future climatic conditions. However, other underlying or confounding causes may give rise to similar trait relationships without representing plant strategies and without resulting in predictive patterns. To date, it remains unknown whether economics spectra reflect plant strategies in reality (see Box 1 for terminology and definitions).

Some studies have argued to disregard LES as general plant strategies. For instance, Osnas et al. (2013) suggested that some of the strong correlations among the above-mentioned six LES traits might be induced by mass normalization and thus might not represent plant strategies (Lloyd et al., 2013), whereas Westoby et al. (2013) and Poorter et al. (2014) emphasized the value of those mass-based LES traits from a carbon investment perspective. Edwards et al. (2014) also questioned if the so-called LES trade-off actually constitutes generic evolutionary trajectories of those traits, as they did not find a correlation between LL and LMA in deciduous species. Moreover, both Dwyer \& Laughlin (2017) and a meta-analysis by Zeballos et al. (2017) found that the correlation between plant traits was context-dependent and that stressful climatic conditions strengthened this relationship. Although this phenomenon may indicate an eco-evolutionary constraint, it also may indicate trait convergence of two otherwise independent traits under the influence of a common driver. Given that water and nutrient availability affect most LES traits, the availability of these resources may act as a common 
environmental driver that leads to trait correlation without necessarily representing a plant strategy.

Other studies interpreted LES as plant strategies by proposing physiological and eco-evolutionary mechanisms and evidence for these LES trait-trait relationships. For example, Shipley et al. (2006) proposed that the LES could be explained by a fundamental trade-off between allocation of plant resources to structural tissues or leaf photosynthetic processes. More recently, Onoda et al. (2017) provided physiological and structural support for this assumption in a meta-analysis based on anatomical and other rarely measured traits. They found that long LLs were achieved by higher LMA and, in turn, by a higher cell wall mass fraction, which inevitably reduced photosynthetic efficiency. In addition, from an eco-evolutionary point of view, Donovan et al. (2011) argued that most of the trait concordance may be caused by selection, which leads to the elimination of low fitness individuals with those leaf trait combinations that function poorly in given biotic and abiotic conditions.

Although extensive research about plant strategies has been carried out, most studies have focused on trait relationships resulting from interspecific variation. Instead, by assessing trait correlations for both intraspecific and interspecific variation, it can be discerned whether LES (PES, or any trait-trait relationship) represents a plant strategy or is a coincidental relationship unlikely to be associated directly with a strategy. Assuming that the LES or PES spectra arise through physiological mechanisms or eco-evolutionary constraints, one would expect that the traittrait relationships observed between species also exists within species. If so, these within-species relationships should express themselves through the relationships of intraspecific trait variation (ITV) among traits, because ITV can be considered as a plant strategy characteristic. Compared with between-species trait-trait relationships, ITV is likely to better reflect trade-offs between traits based on resource allocation constraints. Because, for example, within an individual plant, investing available resources into one trait would directly constrain the investment of remaining resources to other traits (Eichenberg et al., 2015). Evaluating the ITV relationships among traits therefore is probably a more direct assessment of the physiological mechanisms or eco-evolutionary constraints driving trait correlation than assessing the relationships between species alone, for which the likelihood of confounding factors (such as common drivers or differences in habitats) is larger. However, the evidence for traittrait relationships within species is limited, and most studies assessed only a small number of species (e.g. Niinemets, 2015; Martin et al., 2017). The only large-scale analysis (39 paired species for ITV) so far (Anderegg et al., 2018) found mixed evidence for the existence of LES trait correlation within species.

The objective of this paper was to assess whether LES (as well as some PES trait relationships) are plant strategies by comparing the direction and strength of trait-trait relationships within species and between species in a global meta-analysis. In this study, we used the slope of the within-species trait variation vs the community trait variation as a measure of ITV (Ackerly \& Cornwell, 2007; Lepš et al., 2011). Similar to alternative measures of ITV (e.g. the coefficient of variation), this metric is unit- less and thus aids comparison across traits. However, in contrast to alternatives, our metric is not affected by the length of the environmental gradient that is studied nor the absolute variation in trait values, and these influences therefore will not bias reported estimates of ITV or its covariation.

We propose a conceptual framework that illustrates how different relationships (positive, negative and none) in interspecific trait variation translate into a possible correlation of ITVs. Assuming that physiological mechanisms or eco-evolutionary constraints are drivers of both within and between trait-trait relationships, we can compare these relationships to discern between plant strategies and coincident relationships (Fig. 1; see also Box 1 for terminology and definitions). In the case of a plant strategy across species, we will observe either positive or negative traittrait relationships between species means, combined with a positive ITV coordination across species (the variability of the traits within species is positively correlated, which means that plants change both traits at the same time; Fig. 1a,b). This implies that negative ITV correlations (suggesting that high within-species variation within one trait coincides with low within-species variation in another trait) driven by physiological mechanisms or ecoevolutionary constraints across scales should not exist (Fig. 1d,e). If species deal with environmental pressures by alternative adaptation strategies, and thus change either one or another trait, then we would expect to see no relationship between these two traits across species but a negative correlation in ITV (Fig. 1f). Consequently, Fig. 1(c) should not exist. If the trait correlation that is observed between species is strongly caused by common drivers, then there would be no strong correlation between ITVs (Fig. 1g,h). Fig. 1(i) shows that if there is no trait correlation between species because of the absence of a corresponding plant strategy, then we will not observe trait-trait relationship within species either. We evaluated our framework by compiling and analysing a global database of multiple trait data of 2064 species from 19 studies.

\section{Materials and Methods}

\section{Database preparation}

In order to test our framework, we prepared a global database of ITV of multiple traits in four steps. First, we collated published and unpublished datasets for inclusion in a trait database for analysis of ITV. Data were obtained from three sources: unpublished datasets from our previous and current research projects; published data from supplementary materials or public databases where datasets had been deposited; and original datasets of published articles which were directly provided by the authors. To obtain data from the latter sources, we searched Google Scholar using various combinations of the following keywords: plasticity, intraspecific trait variation, intraspecific trait (variation or variability), individual trait, trait gradient analysis, Ackerly \& Cornwell, and Lepš. For resulting studies, we evaluated whether the paper as well as its associated data contained the following information: (1) a list of plots (or communities); (2) a list of species sampled in those plots with at least one species occurring 


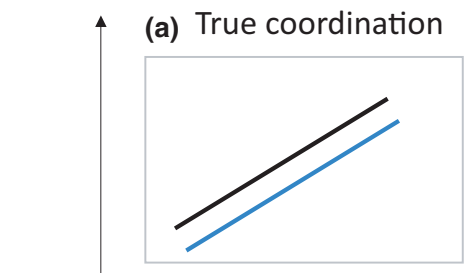

(d)

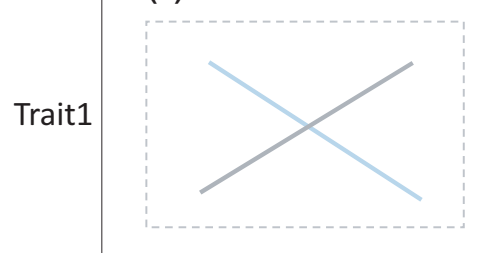

(g) Coincident coordination

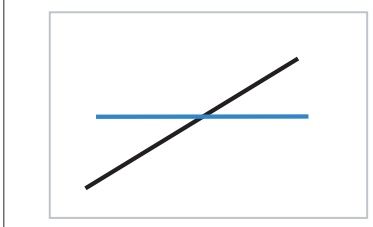

(b) True trade-off

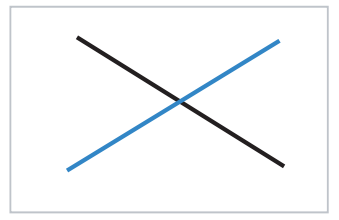

(e)

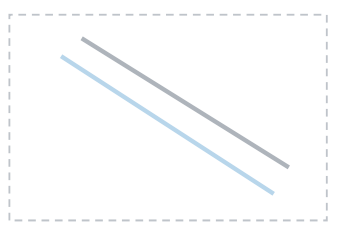

(h) Coincident trade-off

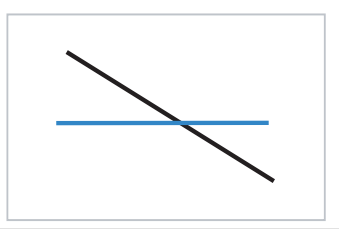

(c)

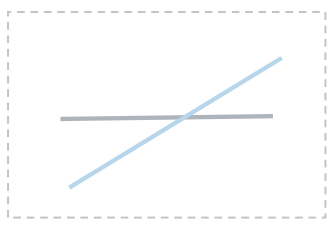

(f) Alternative strategy

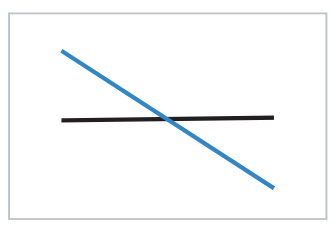

(i) No plant strategy

Trait2

Fig. 1 Hypothetical trait-trait relationships between species (showing interspecific trait variation, in black) and within species (showing intraspecific trait variation (ITV), in blue). (a) True coordination, positive trait-trait relationships both between and within species. (b) True trade-off, negative trait-trait relationship between species but positive within species. Both true coordination and trade-off are considered as plant strategies. (c-e) Do not exist according to our hypotheses. (f) Dominance of alternative strategies, either of the two traits is adapted at a given situation to deal with an environmental pressure. (g) Coincident coordination, positive trait-trait relationships between species but no relationships within species. (h) Coincident trade-off, leading to negative trait-trait relationships between species but no relationships within species. (i) No plant strategy involved in the assessed traits, no trait-trait relationship neither between or within species. See Box 1 for more details on definitions and terminology.

in three or more plots; (3) a list of plot-mean trait values or individual trait values measured for sampled species in each plot; and (4) a list of abundances for each species in each plot (or species richness data of individual plots to allow estimating species abundance). If all four criteria were met, available data were downloaded or authors were contacted for provision of their trait data or complementary data (e.g. abundance data). We also searched published datasets deposited in the Dryad Digital Repository (https://datadryad.org/search) by using the following keywords: individual trait, intraspecific trait. Our trait database was compiled from 17 resulting studies (Ordoñez et al., 2010; Kembel \& Cahill, 2011; Kichenin et al., 2013; Siefert et al., 2014; Carmona et al., 2015; Jager et al., 2015; Buzzard et al., 2016; Cheng et al., 2016; Spasojevic et al., 2016; de la Riva et al., 2016; Derroire et al., 2018; Li et al., 2018; Fajardo \& Siefert, 2019; Niu et al., 2020; E. de Goede et al., unpublished (two datasets); P. M. van Bodegom et al., unpublished; for more details, please see Supporting Information Table S1).

Secondly, we cleaned the data, by removing observations of taxa that had not been classified to species level, omitting observations on mosses, lichens, clubmosses and succulents, and updating species names to accepted names according to The Plant List (http://www.theplantlist.org/) using the $\mathrm{R}$ package
TAXONSTAND. Moreover, we ensured that units of each trait were consistent and created unique plot identification codes (IDs). We unified the abundance of all studies to relative abundance so that the sum of abundances of all species in each plot was $100 \%$. For four studies that provided individual records rather than abundance, we calculated their relative abundance from the number of individuals of each species in the plot divided by the total number of individuals in the plot (Table S1). All trait data were measured in the field, and predominantly on adult individuals. However, a limited number of observations relate to seedlings from tree species. To reduce any ontogenetic bias, we excluded any maximum height observations of tree seedlings from the database.

Thirdly, for each species and each trait in our database, we calculated the magnitude of ITV and species mean trait value using trait-gradient analysis (Ackerly \& Cornwell, 2007; see their section 2.2) and compiled them into a new dataset, together with two datasets (studies 15 and 16 in Table S1) that already contained, for 120 species, ITVs and species mean trait values derived from the trait-gradient analysis (Ackerly \& Cornwell, 2007; Kooyman et al., 2010).

Fourthly, traits which had data from at least four studies were selected, resulting in a dataset of 11 functional traits, namely 
Box 1 Definitions of terms used in this study and mechanisms behind our framework

Plant strategy: There are many different definitions of plant strategies, but all describe how species respond to their environment and recognize that plants have a finite set of resources allocated in different ways to growth, reproduction and maintenance. Functional trait-based strategies relate to a set of different trait values, expressing the investment of individuals to optimize their fitness in a given environment.

In this study, we focus on relationships between two traits. Relationships between traits of different species can show a lack of a significant correlation, a positive correlation ('coordination') or a negative correlation ('trade-off').

An apparent significant coordination or trade-off between two traits of different species may be caused by three mechanisms:

(1) Physiological mechanism, describes the situation in which two correlated traits, because they are linked through chemical and physical plant functions, are limited to a range of values that they can attain. For example, the trade-off between seed quantity and seed mass with species having either a high number of small seeds or a limited number of large seeds because of carbon limitations. Physiological mechanisms lead to 'true tradeoffs' and 'true coordination'. This also includes mathematical dependence among traits, which express physiological dependencies, such as among structural LES traits (see Eqn 4 in main text). We use the word 'true' throughout to refer to trade-offs and coordination where the change of a trait will result in a change of another trait (sensu Grubb, 2016), merely in contrast to 'coincident' relationships, which describe traits that co-vary through a common confounding driver (Mechanism 3 below).

(2) Eco-evolutionary constraint, describes ecological and evolutionary processes that affect the fitness of species, ultimately resulting in the evolution of particular plant strategies. This includes phylogenetic constraint (where possible combinations of trait values have not evolved in a lineage; for example, nitrogen fixation is constrained to legumes) and ecological constraint (where trait combinations are physiologically possible, but lead to lower fitness, which results in the trait combinations being outcompeted, and thus do not lead to an evolutionarily stable strategy). Given that our definitions of coordination and trade-offs do not imply costs, eco-evolutionary constraints can lead to both 'true coordination' and 'true trade-off'. Multiple evolutionary stable strategies may be present in a single environment, as a consequence of multiple eco-evolutionary constraints acting simultaneously, each representing an alternative functional design of approximately equal competence (Marks \& Lechowicz, 2006). Such situations may conceal a 'true coordination' and 'true trade-off'.

(3) Common driver impact, describes a coincident trait-trait relationship that exists as a result of a common environmental driver, which drives the variation of two traits independently without necessarily involving a physiological or eco-evolutionary mechanism that links the two traits. This results in an apparent between-species trait-trait relationship in this study, either 'coincident coordination' or 'coincident trade-off'.

Plant strategies are represented by 'true coordination' and 'true trade-offs' between traits. It is conceptually important to separate the two mechanisms that create these patterns ( 1 and 2 in Box 1), as they have different implications. However, because physiological and eco-evolutionary mechanisms cannot yet be separated empirically, they have been combined in our framework. Using this framework (Fig. 1), we can distinguish plant strategies from coincident relationships. To do so, we jointly assess interspecific trait-trait relationships and the relationships between ITV of those same traits. ITV-ITV relationships represent the trait-trait relationships of ITV, which is equivalent to trait-trait relationships within species.

specific leaf area (SLA), leaf size (LS), leaf dry matter content (LDMC), leaf nitrogen content (LNC), maximum height $(\mathrm{MH})$, leaf phosphorus content (LPC), leaf carbon content (LCC), leaf thickness (Lth), leaf tissue density (Ltis), stem specific density (SSD) and specific root length (SRL). In total, the final ITV database contained 2064 species (Table S2) and these observations came from 1068 communities (plots) across 19 countries covering tropical, temperate and boreal biomes (Fig. 2; Table S1).

\section{Defining intraspecific trait variation using trait-gradient analysis}

We defined and determined estimates of ITV using the traitgradient analysis, as outlined by Ackerly \& Cornwell (2007).

The plot mean trait value, $\overline{p_{j}}$ can be expressed mathematically as:

$\overline{p_{j}}=\frac{\sum_{i=1}^{S} a_{i j} t_{i j}}{\sum_{i=1}^{S} a_{i j}}$

Eqn 1

$\left(\overline{p_{j}}\right.$, abundance-weighted mean trait value across all species in plot $j$ (given that traits may be considered to converge under the influence of environmental pressures, this value represents the position of a plot along the environmental gradient driving this trait (Ackerly \& Cornwell, 2007)); $t_{i j}$, individual species trait value of species $i$ in plot $j$; abundance of species $i$ in plot $j$; and $S$, total number of species in plot $j$.)

The species mean trait value $\overline{t_{i}}$ was calculated as:

$\overline{t_{i}}=\frac{\sum_{j=1}^{P} a_{i j} t_{i j}}{\sum_{j=1}^{P} a_{i j}}$

( $P$, total number of plots where this species occurs). The between-species trait-trait relationships presented in this study refer to relationships between species mean trait values.

In addition to calculating ITV (explained below) and species mean trait values, this method also allows determining species niche breadth, $R_{i}$. This is defined as the range of $\overline{p_{j}}$ that species $i$ occupies along the trait gradient. It is calculated as:

$R_{i}=\max \left(\overline{p_{j}}\right)-\min \left(\overline{p_{j}}\right)$

Eqn 3

The trait-gradient analysis expresses ITV relative to the (community-weighted) trait variation in the community. If one visualizes the variation of individual species trait values $t_{i j}$ vs the plot mean trait values $\overline{p_{j}}$ (Fig. 3), sets of points (grey dots) align vertically at a particular value of $\overline{p_{j}}$ which indicate the species that co-occur in the same plot $j$. A weighted least squares (WLS) 


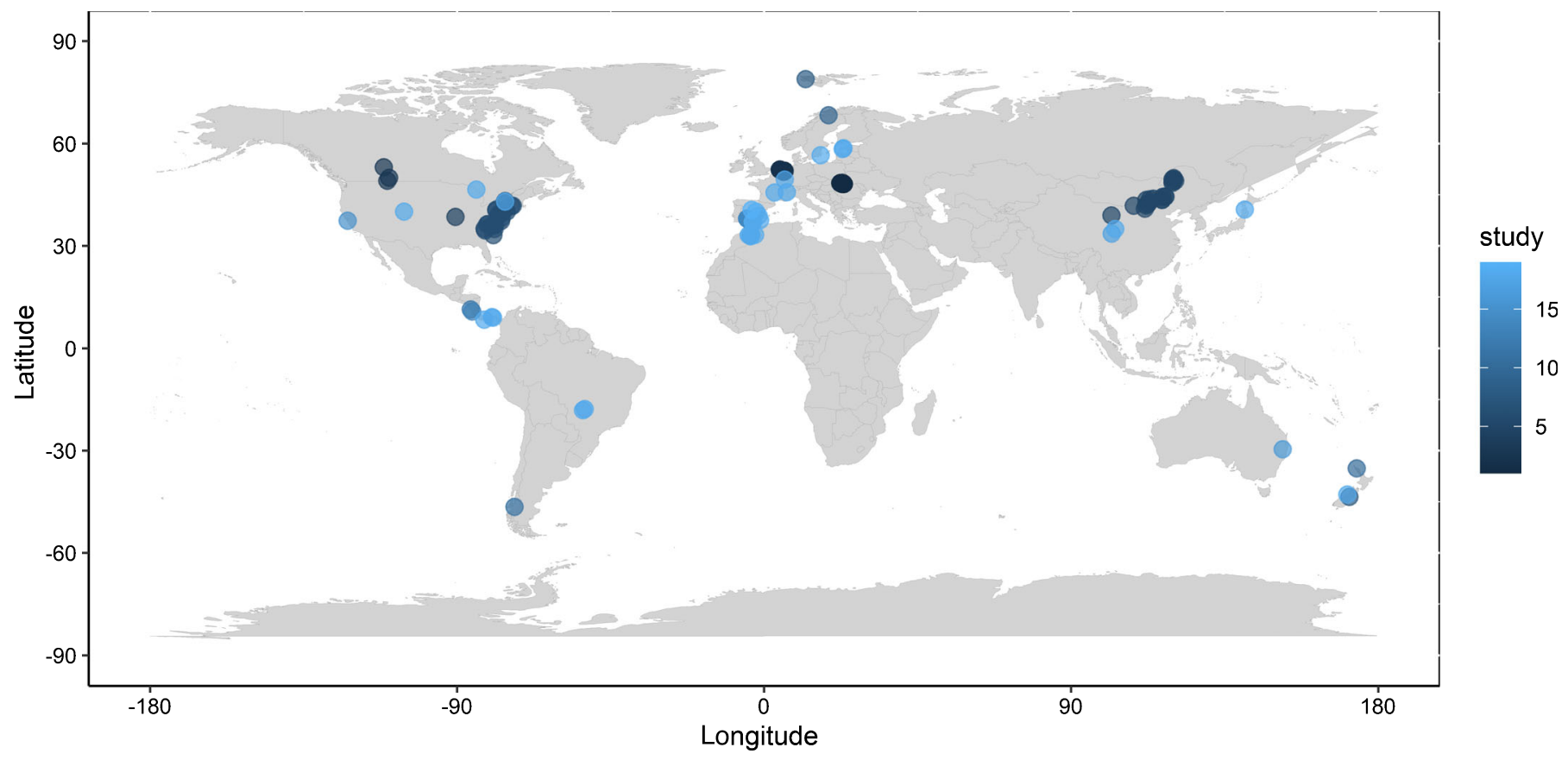

Fig. 2 Site/plot locations of 19 studies which were compiled in our global species intraspecific trait variation database. Some studies contained multiple sites. Those locations were marked in the same colour for each study.

regression through all $t_{i j}$ vs $\overline{p_{j}}$ represents the community trait variation which, by definition, falls on a $1: 1$ line (represented in Fig. 3 by the black dashed line). For an individual species, the slope of the WLS regression line of $t_{i j}$ vs $\overline{p_{j}}$ for that species reflects the magnitude of ITV of that species relative to the community trait variation. Fig. 3 shows an example of the WLS regression for the species Amomyrtus luma (Molina) D. Legrand $\&$ Kausel represented by 134 SLA observations in our global database, weighing each point by plot abundance.

Thus, we express ITV as a slope, instead of expressing intraspecific variation as a percentage of the mean trait value of a given species (e.g. Albert et al., 2010; Messier et al., 2010; Violle et al., 2012). The range over which a species' trait varies is likely to increase with an increase in length of the environmental gradient, which renders the latter approach sensitive to the length of the gradient. Instead, expressing ITV as a slope is much less affected by the length of the environmental gradient for which observations of an individual species are available. At the same time, like other metrics, our ITV metric is unit-less and thus allows direct comparison across traits.

We repeated the trait-gradient analysis procedure to calculate ITVs and species mean trait values for all 11 traits. For most traits, except for LDMC, LCC and SSD, their original trait values did not conform to a normal distribution, thus their $\log _{10^{-}}$ transformed trait values were used in these calculations.

Before testing our hypotheses, we scrutinized the robustness of the ITV values in our database. First, we omitted those species for which estimates of ITV were associated with a large estimation error ( $\mathrm{SE}>1$; our results were robust to choices in the $\mathrm{SE}$ threshold according to a sensitivity analysis; see Figs S1, S2). Because slope estimates based on two points are not reliable, we also excluded those species that were present in fewer than three plots. In addition, we found that estimation errors in ITV decreased with increasing width of observed niche breadth. For consistency, we retained those ITV values whose species niche breadths were $>5 \%$ of the average niche breadth values for all species (for more details, please see Notes S1; Fig. S3).

\section{Standard major axis regression}

We used standard major axis (SMA) regression (Warton et al., 2012) to describe the best-fit lines of global pairwise ITV relationships and to compare those to global between species traittrait relationships. To compare the pairwise intra- and interspecific trait variation not affected by sample size or species selection, we looked at exactly the same dataset for each pair of traits. Depending on the trait combination assessed, our tests were performed on data from between eight to 470 species (of 2064 species). We used the sma() function in the SMART package (Warton et al., 2012) in R software (v.3.6.2, R Development Core Team, 2019) to quantify the slopes with $95 \%$ confidence intervals and their associated coefficient of determination $\left(R^{2}\right)$ of the bivariate relationships for both trait ITVs (the slopes) and species mean trait values $\left(\overline{t_{i}}\right)$ (the interspecific variation) for all pairs of the abovementioned 11 functional traits. We classified our 11 traits into three groups: LES-related traits (SLA, LDMC, LNC, LPC, LCC, Lth, Ltis; further referred to as LES traits), size and structure-related traits (LS, MH, SSD (see Díaz et al., 2016); further referred to as size-related traits) and rootrelated trait (SRL), and describe the results of the correlation of the traits within and between these three groups. In addition, we cluster some of the LES-related traits (SLA, LDMC, Lth and Ltis; together referred to as structural LES traits), given that these traits have a mathematical and possible physiological 


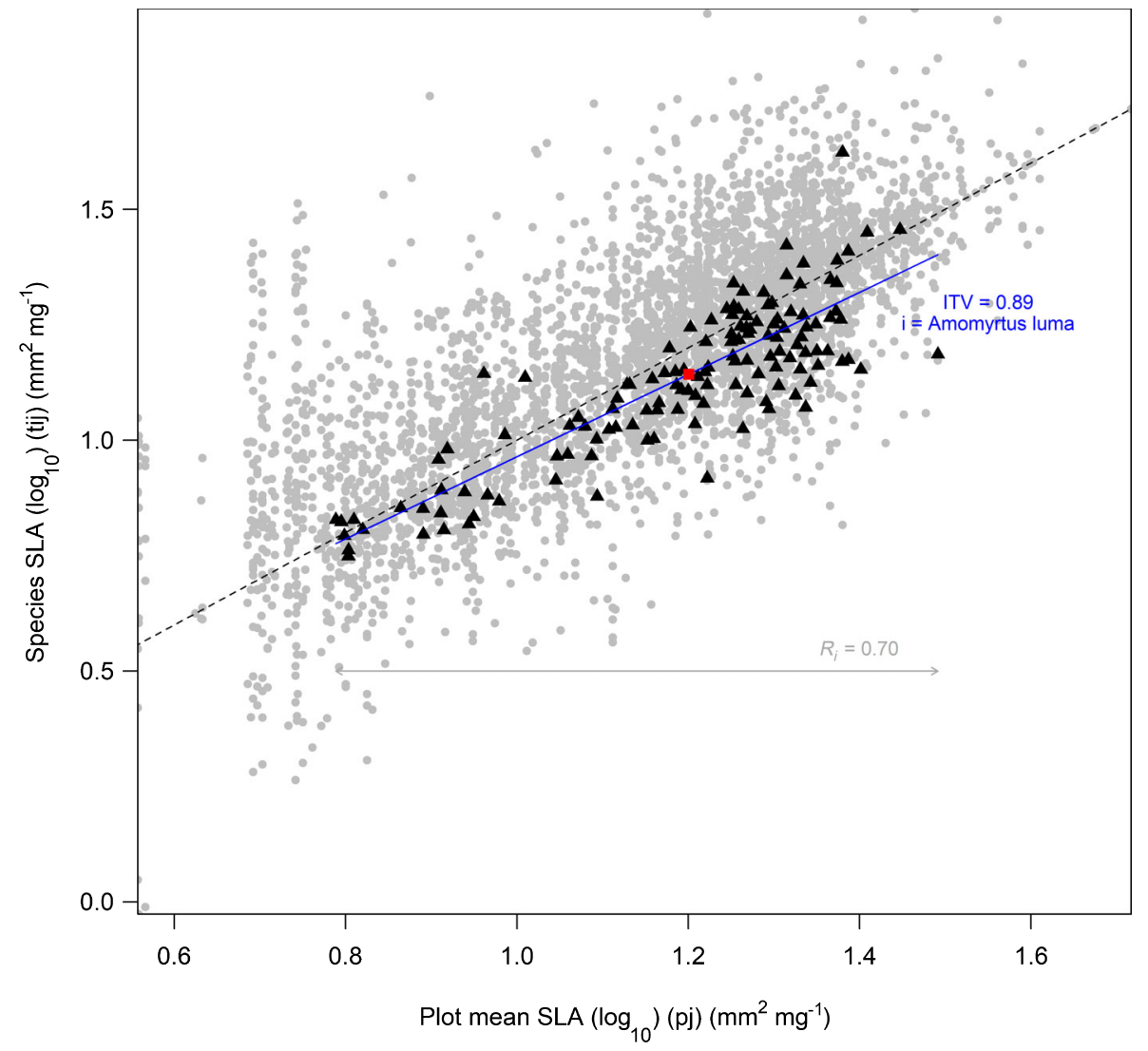

Fig. 3 Scatterplot of individual species trait values $\left(t_{i j}\right)$ vs plot mean trait values $\left(\overline{p_{j}}\right)$ for $\log _{10}$-transformed specific leaf area (SLA) $\left(\mathrm{mm}^{2} \mathrm{mg}^{-1}\right)$ in our trait database. The black dashed line is the $1: 1$ line. Black triangles illustrate the observations of species $i$, in this case Amomyrtus luma. The blue line is the weighted least squares (WLS) regression line for this species and the slope of this line reflects its intraspecific trait variation (ITV $=0.89)$. The red square shows its species mean trait value of $\log _{10}$-transformed SLA $\left(\overline{t_{i}}\right)$ at its position along the plot mean trait gradient expressed by $\log _{10}$-transformed SLA. Grey segment with arrowheads represents the niche breadth $\left(R_{i}=0.70\right)$ of Amomyrtus luma along this trait gradient. See text for further explanation of these parameters. dependency (Pérez-Harguindeguy et al., 2013) according to the following equation:

$\mathrm{LDMC}=\frac{1}{\text { leaf tissue density } \times \text { SLA } \times \text { leaf thickness }} \quad$ Eqn 4

\section{Results}

Trait-trait relationships between species did not necessarily reflect trait-trait relationships within species. Most trait-trait relationships (39 of 54) were significant between species (Table 1). Fifteen of these 39 trait-trait relationships between species remained significant within species, suggesting that they may represent plant strategies according to our framework (Fig. 1a,b). The other 24 trait-trait relationships between species are consistent with coincident coordination or coincident trade-off (Fig. $1 \mathrm{~g}, \mathrm{~h})$. Moreover, although some trait-trait relationships between species had an $R^{2}>0.2$, the strength of these relationships was reduced within species (Table S3). Finally, we did not find any evidence for alternative strategies (Fig. 1f) among the 11 traits under investigation (Table 1).

\section{Leaf economic spectrum traits}

All structural LES traits (SLA, LDMC, Lth, Ltis) were positively related within species. Between species, LDMC was related positively with Lth and Ltis (Fig. 4d,e), whereas all others were correlated negatively (Fig. 4a-c,f). For some trait-trait relationships within species, the strength of the relationship was similar to that of between species (LDMC vs Lth, Lth vs Ltis; Fig. 4d,f). However, generally, within species trait-trait relationships were much weaker than those between species. At the extreme ends, we found SLA vs Ltis (Fig. 4c) with a similar strength of traittrait relationships within and between species $\left(R^{2}=0.194\right.$ and 0.190 , respectively), whereas for SLA vs Lth (Fig. 4b) the correlation within species was a magnitude weaker $\left(R^{2}=0.036\right)$ than between species $\left(R^{2}=0.412\right)$.

Specific leaf area and LDMC and other LES-related traits, LNC, LPC and LCC showed variable correlation within and between species (Fig. 5). SLA showed no within-species relationship with LNC, but a positive one between species (Fig. 5a). LCC showed no relation within species and negative between-species relations with SLA, LPC and LNC (Fig. 5c,h,i), whereas LCC showed positive relations at both scales with LDMC (Fig. 5f). LDMC showed a positive within-species and a negative between-species relationship with both LNC and LPC (Fig. 5d,e). LPC was positively related both within species and between species to LNC and SLA (Fig. 5b, g). For all of these traits, correlation within species was much weaker than that between species, as indicated by the $R^{2}$.

\section{Size-related traits}

Leaf size (LS) did not show within-species correlation with any LES traits (Fig. 6a,c-f), except for a very weak positive 
Table 1 Different mechanisms based on pairwise trait-trait relationships within and between species. Following our framework, the 54 pairwise trait-trait relationships between and within species were classified into different mechanisms (true trade-off/coordination, coincident trade-off/coordination, alternative strategy, or no plant strategy; see Fig. 1). Each row represents a mechanism based on the significance of relationships within and between species. Columns represent different groups of traits: among leaf economic spectrum traits, between LES and size-related traits, among size-related traits, and between root-related and other traits. Each cell indicates trait-trait pairs from each trait group (column) that show a certain mechanism (row). Within each cell, the trait-trait relationships are listed in order of decreasing $R^{2}$ of the between-species relationships. Italicized trait pairs have an $R^{2}<0.2$ for between-species relationships. The three within-species trait-trait significant $(P<0.05)$ relationships are marked in bold $\left(R^{2}\right.$ range $\left.0.183-0.202\right)$.

\begin{tabular}{|c|c|c|c|c|}
\hline Mechanism & Among LES traits & Size vs LES traits & Among size traits & SRL vs other traits \\
\hline \multirow[t]{7}{*}{ True coordination } & LDMC-Ltis & $S S D-L D M C$ & & \\
\hline & LDMC-LCC & & & \\
\hline & LNC-LPC & & & \\
\hline & LCC-Ltis & & & \\
\hline & SLA-LPC & & & \\
\hline & $L C C-L t h$ & & & \\
\hline & LDMC-Lth & & & \\
\hline \multirow[t]{6}{*}{ True trade-off } & SLA-Lth & $L S-L D M C$ & & \\
\hline & LDMC-LPC & & & \\
\hline & SLA-LDMC & & & \\
\hline & LDMC-LNC & & & \\
\hline & Lth-Ltis & & & \\
\hline & SLA-Ltis & & & \\
\hline \multirow[t]{5}{*}{ Coincident coordination } & SLA-LNC & SSD-LCC & $\mathrm{MH}-\mathrm{SSD}$ & $S R L-S L A$ \\
\hline & & $M H-L C C$ & MH-LS & \\
\hline & & LS-LCC & & \\
\hline & & $M H-L D M C$ & & \\
\hline & & $L S-L N C$ & & \\
\hline \multirow[t]{6}{*}{ Coincident trade-off } & SLA-LCC & SSD-SLA & & SRL-MH \\
\hline & LNC-Lth & MH-LPC & & SRL-Lth \\
\hline & LPC-LCC & $M H-S L A$ & & SRL-SSD \\
\hline & LPC-Ltis & $S S D-L P C$ & & \\
\hline & LPC-Lth & LS-Lth & & \\
\hline & $L N C-L C C$ & $S S D-L N C$ & & \\
\hline \multirow[t]{7}{*}{ No plant strategy } & LNC-Ltis & LS-SLA & SSD-LS & SRL-LNC \\
\hline & & LS-LPC & & SRL-LPC \\
\hline & & LS-Ltis & & SRL-LDMC \\
\hline & & MH-Lth & & SRL-LCC \\
\hline & & MH-Ltis & & SRL-Ltis \\
\hline & & SSD-Lth & & SRL-LS \\
\hline & & $\mathrm{MH}-\mathrm{LNC}$ & & \\
\hline Alternative strategy & & & & \\
\hline
\end{tabular}

Trait abbreviations: SLA, specific leaf area; LDMC, leaf dry matter content; LNC, leaf nitrogen content; LPC, leaf phosphorus content; LCC, leaf carbon content; Lth, leaf thickness; Ltis, leaf tissue density; LS, leaf size; MH, maximum height; SSD, stem specific density; SRL, specific root length. Note that the trait pair Ltis-SSD was not assessed owing to a lack of paired data.

coordination with LDMC $\left(R^{2}=0.025\right.$; Fig. 6b). Between species, LS was positively related to LNC and LCC (Fig. 6c,e), negatively related to LDMC and Lth (Fig. 6b,f), and not related to SLA and LPC (Fig. 6a,d). Other size-related traits, SSD and $\mathrm{MH}$, showed similar patterns to those of LS (Table S3). None of the three size-related traits was related to leaf tissue density within or between species (Tables 1; S3).

The three size-related traits (MH, LS and SSD) showed no relationship within species (Fig. 7), whereas $\mathrm{MH}$ had strong association with LS and SSD between species (Fig. 7a,b).

\section{Root-related traits}

For root-related traits, we had sufficient pairwise data only for SRL. Between species, SRL was correlated with four (SLA, MH,
Lth and SSD) of the other 10 traits, but within species it was not related to any of these traits (Tables $1 ;$ S3).

\section{Discussion}

Our study compared trait-trait relationships within and between species to assess whether the commonly reported interspecific trait relationships reflect plant strategies. Using a novel approach and a global dataset of 2064 species and 11 traits, we assessed whether the direction and strength of trait-trait coordination and trade-offs observed between species were still maintained within species. Our conceptual framework (Fig. 1) allowed us to discern between trait-trait relationships that represent plant strategies and those that are generated by coincidental factors. 
(a) $R^{2}=0.116 ; P<0.001 ;(325)$

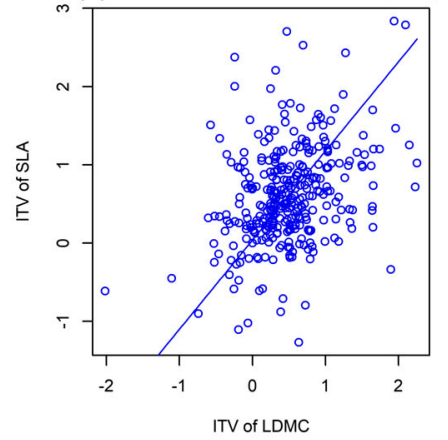

(b) $R^{2}=0.036 ; P=0.017 ;(157)$

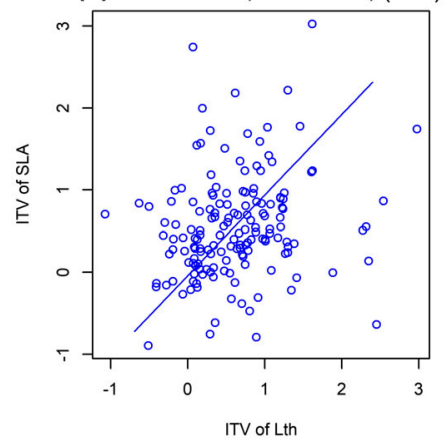

(c) $R^{2}=0.194 ; P<0.001 ;(81)$

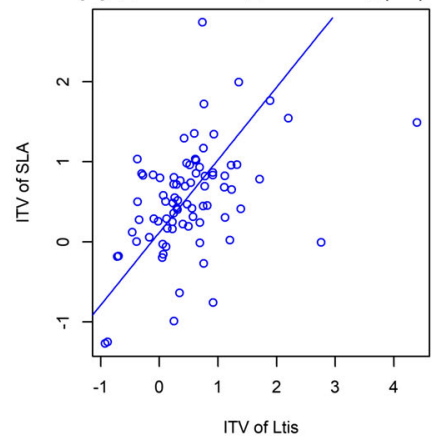

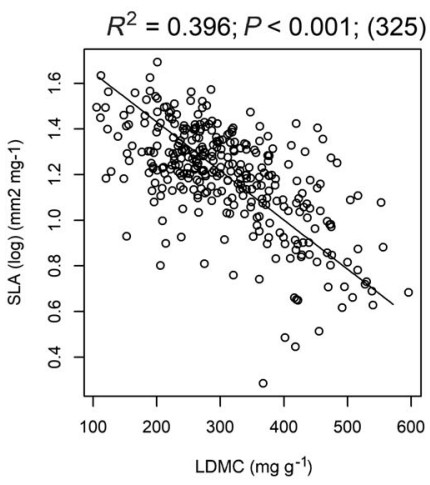
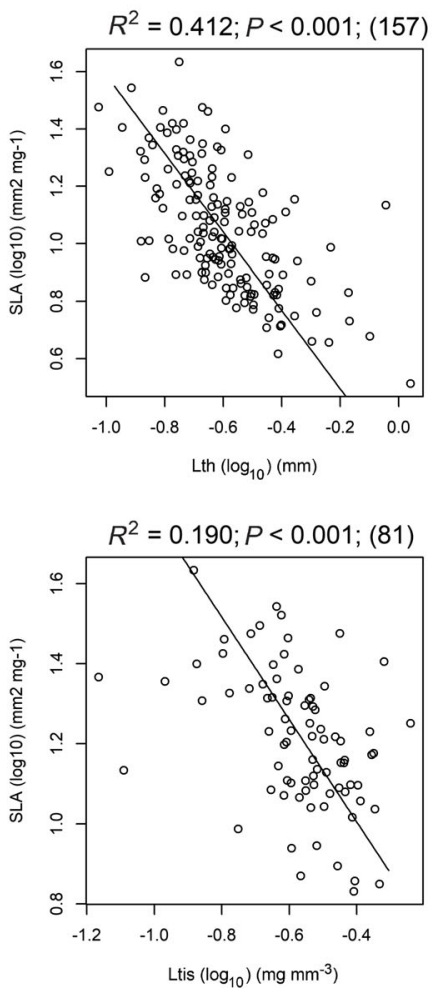

(d) $R^{2}=0.063 ; P=0.025 ;(80)$

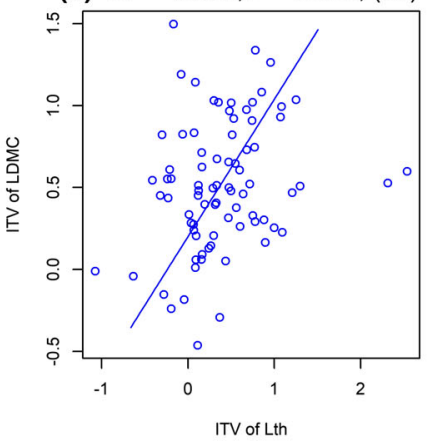

(e) $R^{2}=0.149 ; P=0.002 ;(63)$

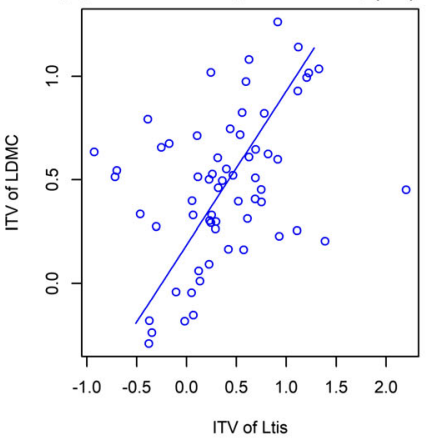

(f) $R^{2}=0.202 ; P<0.001 ;(76)$

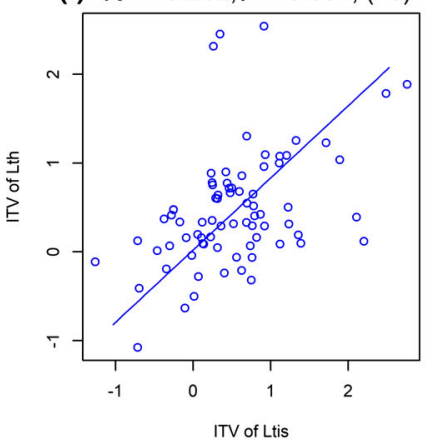

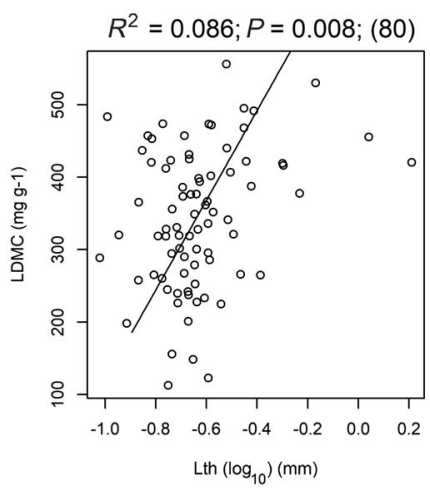
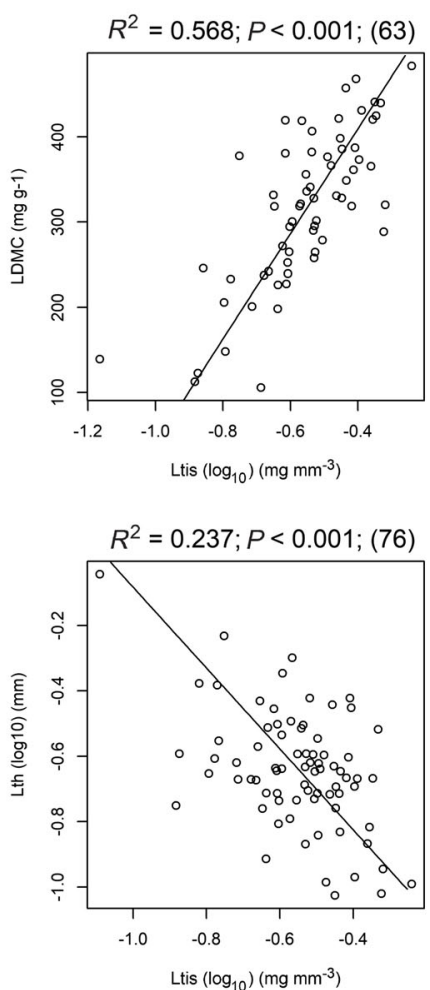

Fig. 4 Relationships among pairs of the structural leaf economic spectrum (LES) traits, specific leaf area (SLA), leaf dry mass content (LDMC), leaf thickness (Lth) and leaf tissue density (Ltis), within species (showing intraspecific trait variation, in blue) and between species (showing interspecific trait variation in black): (a) SLA vs LDMC, (b) SLA vs Lth, (c) SLA vs Ltis, (d) LDMC vs Lth, (e) LDMC vs Ltis and (f) Lth vs Ltis. If a significant relationship was found, the regression line was drawn and the $R^{2}$ was indicated above the figure, followed by the $P$-values. Numbers in brackets refer to the number of species included in the tests.

\section{Overall comparison patterns}

Our conceptual framework (Fig. 1) identified trait pairs that represented true coordination or trade-offs that may represent plant strategies (first and second rows of Table 1). It also identified those trait pairs that expressed coincident coordination or tradeoffs which might mostly be driven by common environmental drivers (third and fourth rows of Table 1).

As expected from our framework, true coordination or tradeoff between species translated into positive within-species (ITV) relationships of corresponding traits. Moreover, those trait pairs that were not related between species, did not show any relationship within species either. We can therefore confirm that those trait pairs are not correlated at any scale (fifth row of Table 1).
We did not find any evidence for our hypothesized occurrence of alternative strategies (sixth row of Table 1). This suggests that, if alternative strategies do occur, different species might not have the same strategy to deal with environmental pressure. This is in line with theoretical studies which suggested that alternative strategies can be expressed in multiple trait combinations (Marks \& Lechowicz, 2006).

\section{Nonconsistent patterns among different trait groups}

Building on previously presented global analyses (Wright et al., 2004; Díaz et al., 2016), we expected that our approach would identify trait-trait relationships caused by plant strategies at least within the same trait group (LES and size-related trait 

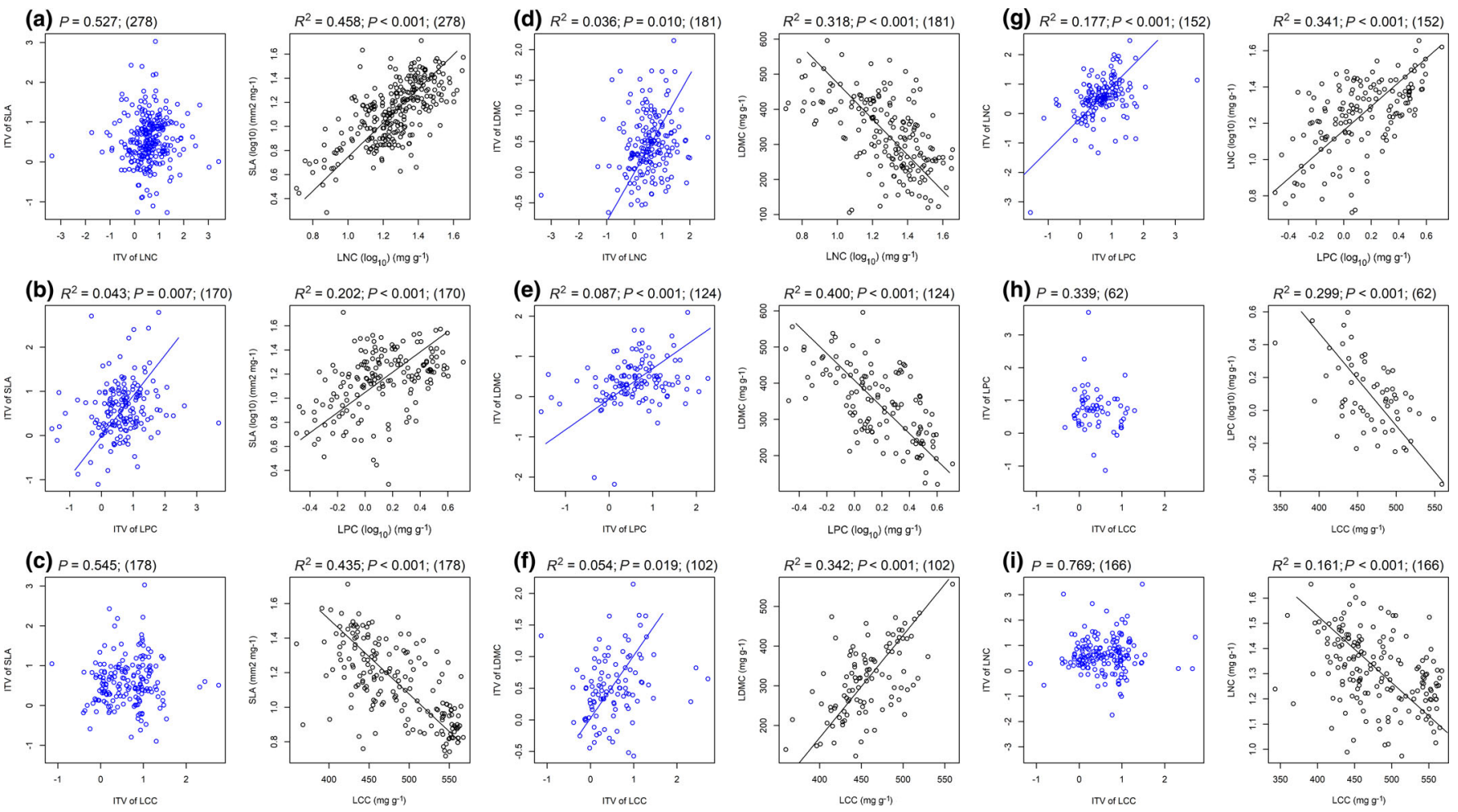

Fig. 5 Relationships among pairs of other leaf economic spectrum (LES)-related traits, specific leaf area (SLA), leaf dry matter content (LDMC), leaf nitrogen content (LNC), leaf phosphorus content (LPC) and leaf carbon content (LCC), within species (showing intraspecific trait variation, in blue) and between species (showing interspecific trait variation in black): (a) SLA vs LNC, (b) SLA vs LPC, (c) SLA vs LCC, (d) LDMC vs LNC, (e) LDMC vs LPC, (f) LDMC vs LCC, (g) LNC vs LPC, (h) LPC vs LCC and (i) LNC vs LCC. If a significant relationship was found, the regression line was drawn and the $R^{2}$ was indicated above the figure, followed by the $P$-values. Numbers in brackets refer to the number of species included in the tests.

groups). Although it was true for LES traits, this was not the case in general. Within LES traits, there were many consistently significant trait-trait relationships at both between- and within-species scales. Consistently significant patterns were especially common for structural LES traits (Fig. 4), for which all significant trait-trait relationships between species were maintained within species. This pattern may be a consequence of the mathematical and possibly physiological dependency among these traits (Pérez-Harguindeguy et al., 2013, see Materials and Methods).

For other LES-related traits, we noticed that both SLA and LDMC were closely associated with LNC, LPC and LCC between species (Fig. $5 \mathrm{a}-\mathrm{f}$ ), whereas within species LDMC was related more strongly (higher $R^{2}$ ) to LNC, LPC and LCC than was SLA. This pattern may be related to the role of LDMC in protecting leaves against physical or herbivory damage. High LDMC species tend to have more complex carbon compounds such as lignin in their leaves to protect them against herbivory (Pérez-Harguindeguy et al., 2013), whereas low LDMC leaves are more palatable. Both LNC and LPC are considered to be important traits related to photosynthesis, and they were highly coordinated with each other both within and between species (Fig. 5g).

By contrast, relationships between the three size-related traits found between species disappeared when their within-species patterns were assessed (Fig. 7). This suggests that these traits may have different drivers, which may co-occur at large spatial scales, while being decoupled at the smaller spatial scales in which individual species prevail. For example, we found the apparent coordination of MH and SSD between species (Fig. 7b) was not because they were physiologically or eco-evolutionarily related (as these traits were not related within species). Instead, this between-species correlation was driven by a clustering of nonwoody species at low $\mathrm{MH}$ and low SSD, whereas woody species clustered at a combination of high $\mathrm{MH}$ and high SSD (see Fig. S4), consistent with previous results (Díaz et al., 2016, their Extended Data figs 3a, 4). The nonsignificant relationship between $\mathrm{MH}$ and SSD within species suggests that the variation of $\mathrm{MH}$ might be driven by light competition (Douma et al., 2012), whereas the variation of SSD may be more related to cavitation protection than mechanical support (Hacke et al., 2001; Sperry et al., 2006).

Between size-related traits and LES traits, some statistically significant relationships between species occurred, which is to a large extent also consistent with the findings of Díaz et al. (2016), but these relationships were mostly absent within species (Fig. 6a,c-f; Table 1). These patterns of coincident coordination and trade-off may indicate different drivers for between-species vs within-species trait relationships for those trait pairs. For example, Wright et al. (2017) showed that on a global scale, LS was dominantly affected by latitudinal and 
(a) $P=0.070 ;(470)$

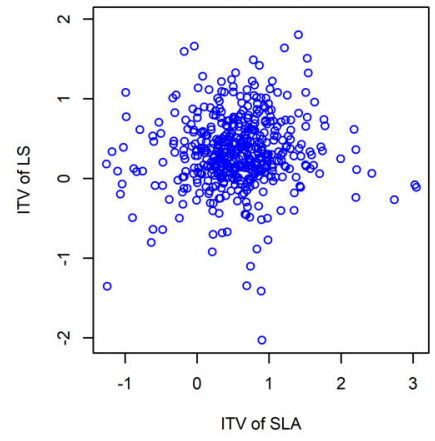

(b) $R^{2}=0.025 ; P=0.029 ;(192)$

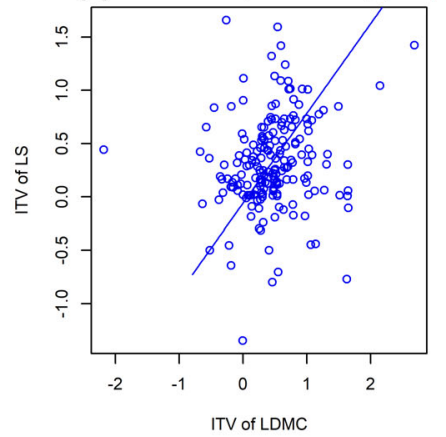

(c) $P=0.552 ;(234)$

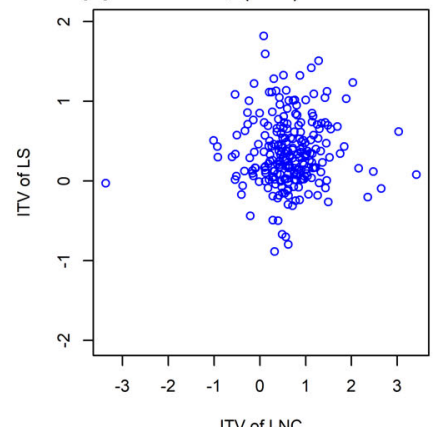

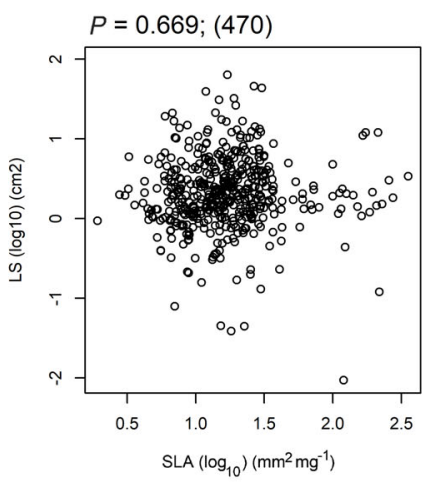
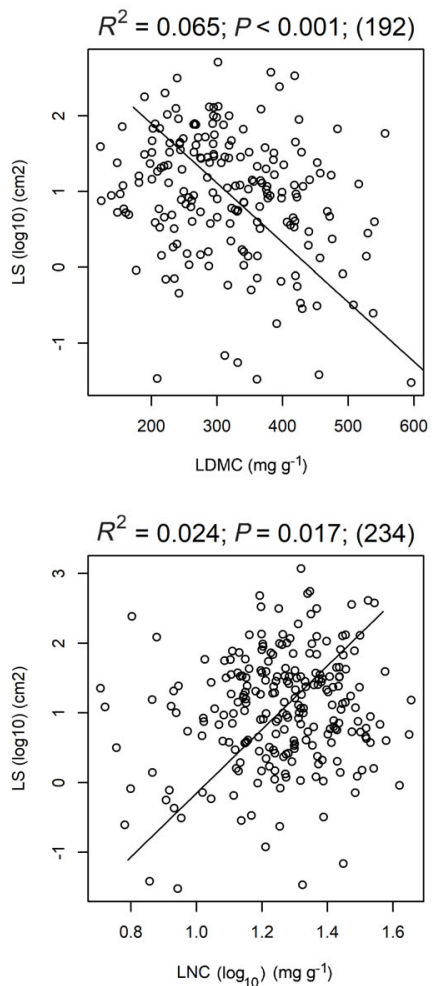

(d) $P=0.914$; (123)

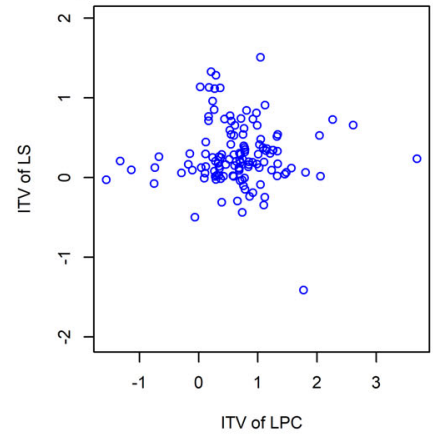

(e) $P=0.127 ;(174)$

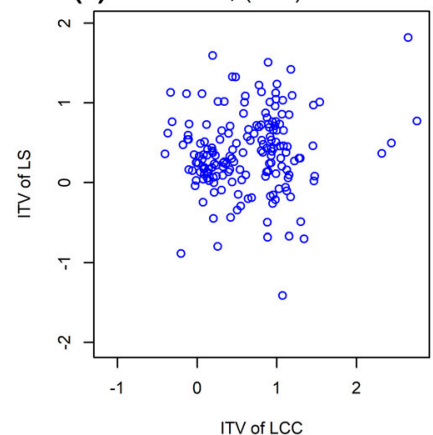

(f) $P=0.709$; (149)

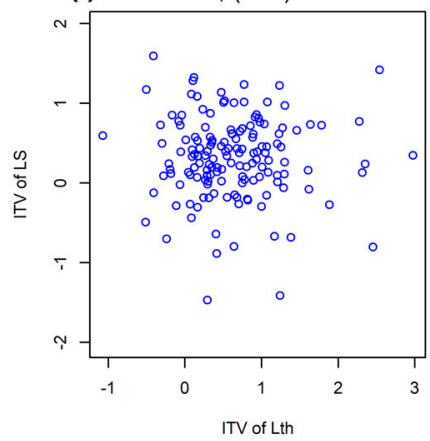

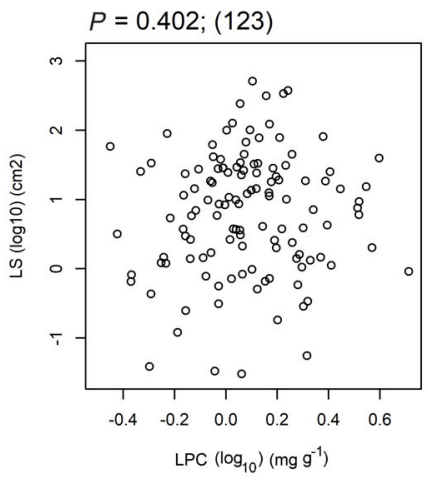
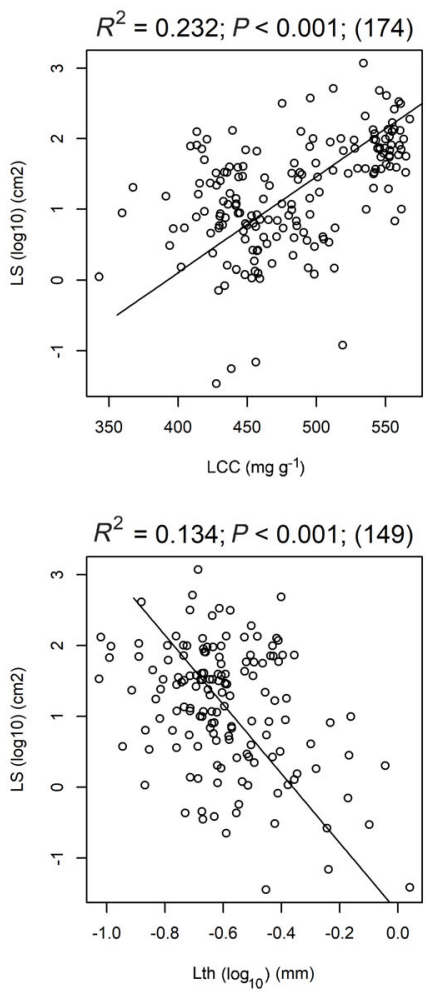

Fig. 6 Relationships of leaf size (LS) to some leaf economic spectrum (LES)-related traits (specific leaf area (SLA), leaf dry matter content (LDMC), leaf nitrogen content (LNC), leaf phosphorus content (LPC) and leaf carbon content (LCC) and leaf thickness (Lth)) within species (showing intraspecific trait variation, in blue) and between species (showing interspecific trait variation in black): (a) LS vs SLA, (b) LS vs LDMC (c) LS vs LNC, (d) LS vs LPC, (e) LS vs LCC and (f) LS vs Lth. If a significant relationship was found, the regression line was drawn and the $R^{2}$ indicated above the figure, followed by the $P$-values. Numbers in brackets refer to the number of species included in the tests.

elevational gradients with different climatic conditions, whereas at local scales LS also was influenced by various other drivers such as plant architecture, canopy display, plant hydraulics, soil fertility and herbivory. These findings may explain why LS was almost unrelated to any LES trait within species in this study.

Although the trait-trait relationships within and between species were generally strongest within the LES traits, we included additional traits to show the general applicability of our approach. For example, we did not find relationships between SRL and any of the LES-related and size-related traits within species. Although the low sample sizes for SRL (compared to the easier to measure LES and size-related traits) may partly have caused the lack of trends, it also may be due to the ITV of SRL varying in species-specific ways along complex environmental gradients (Weemstra et al., 2021). This analysis of traits not strictly associated with LES, such as SRL, $\mathrm{MH}$ and SSD, helps to gain insight into the plant economics spectrum and the extents to and ways in which other traits are independent of the leaf economics spectrum.

\section{Implications}

Our study showed that many of the well-founded trait-trait relationships that occur between species became weaker (as shown by $R^{2}$ values) within species or even disappeared altogether. In fact, except for the SSD-LDMC relationship (Table $1, R^{2}=0.095$ ), 
(a) $P=0.514 ;(224)$

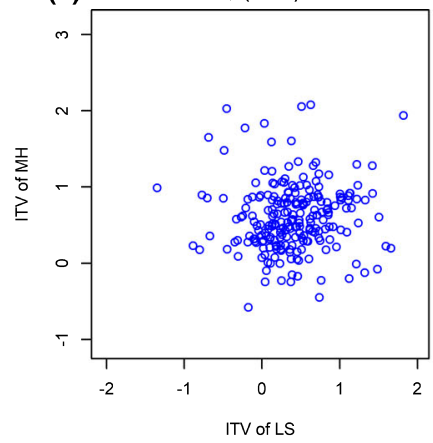

(c) $P=0.733 ;(104)$

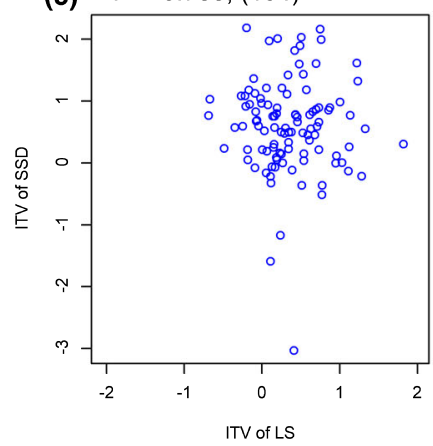

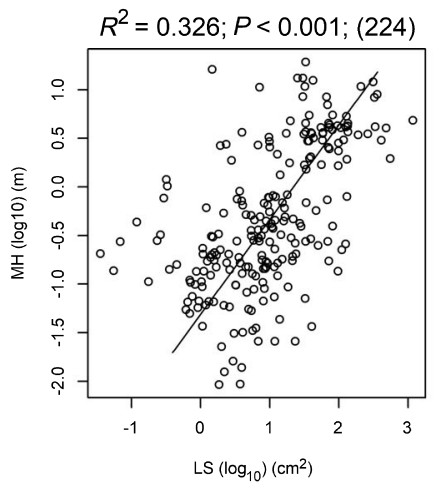
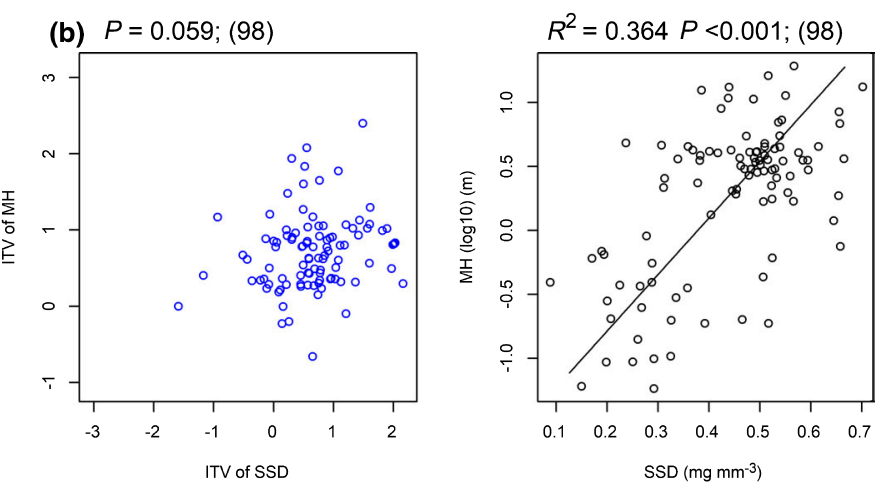

Fig. 7 Relationships among pairs of the size-related traits, maximum height (MH), leaf size (LS) and stem specific density (SSD), within species (showing intraspecific trait variation, in blue) and between species (showing species mean trait values in black): (a) MH vs LS, (b) MH vs SSD and (c) SSD vs LS. If a significant relationship was found, the regression line was drawn and the $R^{2}$ indicated above the figure, followed by the $P$-values. Numbers in brackets refer to the number of species included in the tests.

almost only trait-trait relationships internal to LES-related traits were maintained at the within-species level. Thus, a substantial number of trait-trait relationships previously coined as trade-offs or synergies appear not to indicate plant strategies in reality. Instead, it seems that various between-species trait-trait relationships are the result of co-varying environmental drivers across large scales that may disappear within species. We expect coincident relationships with common environmental drivers to be apparent particularly along large environmental gradients across species (where effects of common drivers become larger than impacts of alternative plant strategies which would reduce the strength of the relationship). Within a species, such relationships would be easy to break because there are no fitness costs involved if the common drivers do not cause true coordination or tradeoffs. Therefore, we would expect to see such trait-trait relationships between species but not within species.

There are several implications for future research based on our findings. We noted that trait-trait relationships within species were weak (the three strongest $R^{2} \approx 0.2$; Table 1 ), which meant that within species, $>80 \%$ of the variation of one trait was decoupled from the variation of other traits. Therefore, to improve our understanding on the role of intraspecific trait variation in plant strategies, it will be important to further investigate the drivers of ITV and its coupling among traits. Importantly, we did not observe any phylogenetic clustering in trait-trait relationships within species (see Figs S5-S9). Hence, we assume that physiological mechanisms and ecological constraints may play more important roles in driving plant strategies within species, than phylogenetic constraints, but this remains to be tested further.

Overall, distinguishing the mechanisms of trait correlation, as we did in this paper, is important for trait predictions, for example under the influence of climate change. If in a future climate, environmental drivers become decoupled, for instance as a result of the rise of no-analogue climatic conditions, this also may lead to the breakdown of some trait-trait relationships between species. Using our findings, we speculate that such decoupling might occur particularly within the so-called sizerelated group of trait variation. Given that many of the traits investigated here are directly related to ecosystem processes, it stands to reason that the expression of some ecosystem processes also are affected. This will be important to account for in model projections.

Our novel conceptual framework (Fig. 1) can be used for further detailed study of extended species and trait sets. Although our newly compiled database already included $>2000$ species and 11 traits in tropical, temperate and boreal communities, much smaller datasets were available for any trait pair. A truly global concerted effort to compile a consistent and larger dataset of within- and between-species trait data will allow a similar approach to further elucidate the mechanisms of general plant strategies and incorporation in ecosystem modelling. 


\section{Conclusions}

Our study describes a novel approach to differentiate trait-trait relationships which may represent plant strategies from those resulting from common environmental drivers. By comparing trait-trait relationships between species vs within species using a unique global database on intraspecific trait variation, we showed that almost only within the leaf economics spectrum, the between-species trait-trait relationships may represent plant strategies. Moreover, the frequent coincidental trait-trait relationships between species, caused by common drivers, imply that model projections on ecosystem functioning in a future climate should seriously consider decoupling of these trait-trait relationships. Our study emphasizes the importance of describing the mechanisms behind trait-trait relationships, both between and within species, to deepen our understanding of general plant strategies.

\section{Acknowledgements}

JZ was funded by a $\mathrm{PhD}$ scholarship from China Scholarship Council (CSC, no. 201608310102). We thank Daniel C. Laughlin, Eefje de Goede, Grégoire T. Freschet and Robert Kooyman for providing the data we needed to expand our database, and Yuanzhi Li and Bill Shipley for clarifying some details of their database when we compiled some of their datasets into our database. We also thank Elise Arnst from the New Zealand National Vegetation Survey (NVS) databank for helping to assess datasets and the support from TRY plant trait database for the categorical databases. We thank Laura Trapero Llousas and Bregje Brinkmann for their assistance with the $\mathrm{R}$ script, and Lisette van Hulst, Amie E. Corbin and Qi Chen for their insights to improve this manuscript. Lastly, we thank the editor and anonymous reviewers for their constructive suggestions which significantly improved the manuscript.

\section{Author contributions}

$\mathrm{PMvB}$ conceived the study; JZ, EC and PMvB developed the ideas; $\mathrm{PMvB}$ and JZ collected the data; JZ, EC and PMvB conducted the analysis; and JZ wrote the first draft. All authors contributed critically to the drafts and gave final approval for publication.

\section{ORCID}

Ellen Cieraad (iD https://orcid.org/0000-0002-9813-9590

Jianhong Zhou (ID https://orcid.org/0000-0001-6347-4498

Peter M. van Bodegom (D) https://orcid.org/0000-0003-07714500

\section{Data availability}

The data that supports the findings of this study are openly available in the Zenodo repository: https://doi.org/10.5281/zenodo. 5684291. Code availability: the R code used to build up the ITV database and generate the results of this study is openly available in the Zenodo repository: https://doi.org/10.5281/zenodo. 5680090 .

\section{References}

Ackerly DD, Cornwell WK. 2007. A trait-based approach to community assembly: partitioning of species trait values into within- and amongcommunity components. Ecology Letters 10: 135-145.

Albert CH, Thuiller W, Yoccoz NG, Soudant A, Boucher F, Saccone P, Lavorel S. 2010. Intraspecific functional variability: extent, structure and sources of variation. Journal of Ecology 98: 604-613.

Anderegg LDL, Berner LT, Badgley G, Sethi ML, Law BE, HilleRisLambers J. 2018. Within-species patterns challenge our understanding of the leaf economics spectrum. Ecology Letters 21: 734-744.

Buzzard V, Hulshof CM, Birt T, Violle C, Enquist BJ. 2016. Re-growing a tropical dry forest: functional plant trait composition and community assembly during succession. Functional Ecology 30: 1006-1013.

Carmona CP, Rota C, Azcárate FM, Peco B. 2015. More for less: sampling strategies of plant functional traits across local environmental gradients. Functional Ecology 29: 579-588.

Cheng J, Chu P, Chen D, Bai Y. 2016. Functional correlations between specific leaf area and specific root length along a regional environmental gradient in inner Mongolia grasslands. Functional Ecology 30: 985-997.

Derroire G, Powers JS, Hulshof CM, Cárdenas Varela LE, Healey JR. 2018. Contrasting patterns of leaf trait variation among and within species during tropical dry forest succession in Costa Rica. Scientific Reports 8: 285295.

Díaz S, Kattge J, Cornelissen JHC, Wright IJ, Lavorel S, Dray S, Reu B, Kleyer M, Wirth C, Colin Prentice I et al. 2016. The global spectrum of plant form and function. Nature 529: 167-171.

Donovan LA, Maherali H, Caruso CM, Huber H, de Kroon H. 2011. The evolution of the worldwide leaf economics spectrum. Trends in Ecology and Evolution 26: 88-95.

Douma JC, de Haan MWA, Aerts R, Witte JPM, van Bodegom PM. 2012. Succession-induced trait shifts across a wide range of NW European ecosystems are driven by light and modulated by initial abiotic conditions. Journal of Ecology 100: 366-380.

Dwyer JM, Laughlin DC. 2017. Constraints on trait combinations explain climatic drivers of biodiversity: the importance of trait covariance in community assembly. Ecology Letters 20: 872-882.

Edwards EJ, Chatelet DS, Sack L, Donoghue MJ. 2014. Leaf life span and the leaf economic spectrum in the context of whole plant architecture. Journal of Ecology 102: 328-336.

Eichenberg D, Purschke O, Ristok C, Wessjohann L, Bruelheide H. 2015. Trade-offs between physical and chemical carbon-based leaf defence: of intraspecific variation and trait evolution. Journal of Ecology 103: 1667-1679.

Fajardo A, Siefert A. 2019. The interplay among intraspecific leaf trait variation, niche breadth and species abundance along light and soil nutrient gradients. Oikos 128: 881-891.

Freschet GT, Cornelissen JHC, van Logtestijn RSP, Aerts R. 2010. Evidence of the 'plant economics spectrum' in a subarctic flora. Journal of Ecology 98: 362373.

Grubb PJ. 2016. Trade-offs in interspecific comparisons in plant ecology and how plants overcome proposed constraints. Plant Ecology and Diversity 9: 3-33.

Hacke UG, Sperry JS, Pockman WT, Davis SD, McCulloh KA. 2001. Trends in wood density and structure are linked to prevention of xylem implosion by negative pressure. Oecologia 126: 457-461.

Jager MM, Richardson SJ, Bellingham PJ, Clearwater MJ, Laughlin DC. 2015. Soil fertility induces coordinated responses of multiple independent functional traits. Journal of Ecology 103: 374-385.

Kembel SW, Cahill JF Jr. 2011. Independent evolution of leaf and root traits within and among temperate grassland plant communities. PLoS One 6 : e19992.

Kichenin E, Wardle DA, Peltzer DA, Morse CW, Freschet GT. 2013. Contrasting effects of plant inter- and intraspecific variation on community- 
level trait measures along an environmental gradient. Functional Ecology 27: $1254-1261$.

Kooyman R, Cornwell W, Westoby M. 2010. Plant functional traits in Australian subtropical rain forest: partitioning within-community from crosslandscape variation. Journal of Ecology 98: 517-525.

Lepš J, de Bello F, Šmilauer P, Doležal J. 2011. Community trait response to environment: disentangling species turnover vs intraspecific trait variability effects. Ecography 34: 856-863.

Li Y, Shipley B, Price JN, Dantas VDL, Tamme R, Westoby M, Siefert A, Schamp BS, Spasojevic MJ, Jung V et al. 2018. Habitat filtering determines the functional niche occupancy of plant communities worldwide. Journal of Ecology 106: 1001-1009.

Lloyd J, Bloomfield K, Domingues TF, Farquhar GD. 2013. Photosynthetically relevant foliar traits correlating better on a mass vs an area basis: of ecophysiological relevance or just a case of mathematical imperatives and statistical quicksand? New Phytologist 199: 311-321.

Marks CO, Lechowicz MJ. 2006. Alternative designs and the evolution of functional diversity. American Naturalist 167: 55-66.

Martin AR, Rapidel B, Roupsard O, Van den Meersche K, Melo Virginio Filho E, Barrios M, Isaac ME. 2017. Intraspecific trait variation across multiple scales: the leaf economics spectrum in coffee. Functional Ecology 31: 604-612.

Messier J, McGill BJ, Lechowicz MJ. 2010. How do traits vary across ecological scales? A case for trait-based ecology. Ecology Letters 13: 838-848.

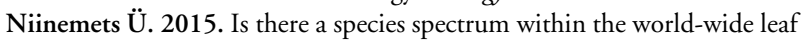
economics spectrum? Major variations in leaf functional traits in the Mediterranean sclerophyll Quercus ilex. New Phytologist 205: 79-96.

Niu K, Zhang S, Lechowicz MJ. 2020. Harsh environmental regimes increase the functional significance of intraspecific variation in plant communities. Functional Ecology 34: 1666-1677.

Onoda Y, Wright IJ, Evans JR, Hikosaka K, Kitajima K, Niinemets U, Poorter H, Tosens T, Westoby M. 2017. Physiological and structural tradeoffs underlying the leaf economics spectrum. New Phytologist 214: 1447-1463.

Ordoñez JC, Van Bodegom PM, Witte JPM, Bartholomeus RP, Van Dobben HF, Aerts R. 2010. Leaf habit and woodiness regulate different leaf economy traits at a given nutrient supply. Ecology 91: 3218-3228.

Osnas JLD, Lichstein JW, Reich PB, Pacala SW. 2013. Global leaf trait relationships: mass, area, and the leaf economics spectrum. Science 340: 741744.

Pérez-Harguindeguy N, Díaz S, Garnier E, Lavorel S, Poorter H, Jaureguiberry P, Bret-Harte MS, Cornwell WK, Craine JM, Gurvich DE et al. 2013. New handbook for standardised measurement of plant functional traits worldwide. Australian Journal of Botany 61: 167-234.

Poorter H, Lambers H, Evans JR. 2014. Trait correlation networks: a wholeplant perspective on the recently criticized leaf economic spectrum. New Phytologist 201: 378-382.

R Development Core Team. 2019. R: a language and environment for statistical computing. Vienna, Austria: R Foundation for Statistical Computing.

Reich PB. 2014. The world-wide 'fast-slow' plant economics spectrum: a traits manifesto. Journal of Ecology 102: 275-301.

de la Riva EG, Pérez-Ramos IM, Tosto A, Navarro-Fernández CM, Olmo M, Marañón T, Villar R. 2016. Disentangling the relative importance of species occurrence, abundance and intraspecific variability in community assembly: a trait-based approach at the whole-plant level in Mediterranean forests. Oikos 125: 354-363.

Shipley B, Lechowicz MJ, Wright I, Reich PB. 2006. Fundamental trade-offs generating the worldwide leaf economics spectrum. Ecology 87: 535-541.

Siefert A, Fridley JD, Ritchie ME. 2014. Community functional responses to soil and climate at multiple spatial scales: when does intraspecific variation matter? PLoS One 9: e111189.

Spasojevic MJ, Turner BL, Myers JA. 2016. When does intraspecific trait variation contribute to functional beta-diversity? Journal of Ecology 104: 487-496.

Sperry JS, Hacke UG, Pittermann J. 2006. Size and function in conifer tracheids and angiosperm vessels. American Journal of Botany 93: 1490-1500.

Violle C, Enquist BJ, McGill BJ, Jiang L, Albert CH, Hulshof C, Jung V, Messier J. 2012. The return of the variance: intraspecific variability in community ecology. Trends in Ecology and Evolution 27: 244-252.
Warton DI, Duursma RA, Falster DS, Taskinen S. 2012. smatr 3- an R package for estimation and inference about allometric lines. Methods in Ecology and Evolution 3: 257-259.

Weemstra M, Freschet GT, Stokes A, Roumet C. 2021. Patterns in intraspecific variation in root traits are species-specific along an elevation gradient. Functional Ecology 35: 342-356.

Westoby M, Reich PB, Wright IJ. 2013. Understanding ecological variation across species: area-based vs mass-based expression of leaf traits. New Phytologist 199: 322-323.

Wright IJ, Dong N, Maire V, Prentice IC, Westoby M, Díaz S, Gallagher RV, Jacobs BF, Kooyman R, Law EA et al. 2017. Global climatic drivers of leaf size. Science 357: 917-921.

Wright IJ, Reich PB, Westoby M, Ackerly DD, Baruch Z, Bongers F, Cavender-Bares J, Chapin T, Cornelissen JHC, Diemer M et al. 2004. The worldwide leaf economics spectrum. Nature 428: 821-827.

Zeballos SR, Giorgis MA, Cabido M, Gurvich DE. 2017. Unravelling the coordination between leaf and stem economics spectra through local and global scale approaches. Austral Ecology 42: 394-403.

\section{Supporting Information}

Additional Supporting Information may be found online in the Supporting Information section at the end of the article.

Fig. S1 Standard error of intraspecific trait variation (ITV) of specific leaf area (SLA) vs ITV of SLA.

Fig. S2 Relationships between SLA and leaf dry matter content (LDMC) within (showing ITV, in blue) and between species (showing interspecific trait variation in black) under different SE thresholds: (a) 4, (b) 3, (c) 2, (d) 1, (e) 0.75 and (f) 0.5.

Fig. S3 The relationships of SE of ITV of SLA vs Ri of $\log _{10^{-}}$ transformed SLA (a) and ITV of SLA vs Ri of $\log _{10}$-transformed SLA (b).

Fig. S4 Relationship between maximum height (MH) and stem specific density (SSD) within species (left) and between species (right). Blue, nonwoody species; red, woody species.

Fig. S5 Within-species trait-trait relationships among LDMC, leaf phosphorus content (LPC), leaf thickness (Lth) and SLA within seven major families: (a) LDMC vs SLA, (b) LPC vs SLA and (c) Lth vs SLA.

Fig. S6 Within-species trait-trait relationships among leaf tissue density (Ltis), SLA, leaf nitrogen content (LNC), leaf phosphorus content (LPC) and LDMC within seven major families: (a) Ltis vs SLA, (b) LNC vs LDMC and (c) LPC vs LDMC.

Fig. S7 Within-species trait-trait relationships among SSD, LDMC, LS and LCC within seven major families: (a) SSD vs LDMC, (b) LS vs LDMC, (c) LCC vs LDMC.

Fig. S8 Within-species trait-trait relationships among Lth, LDMC, Ltis, LPC and LNC within seven major families: (a) Lth vs LDMC, (b) Ltis vs LDMC and (c) LPC vs LNC. 
Fig. S9 Within-species trait-trait relationships among Lth, LCC and Ltis within seven major families: (a) Lth vs LCC, (b) Ltis vs LCC and (c) Ltis vs Lth.

Notes S1 Additional methodological details on data quality control procedures of intraspecific trait variation values.

Table S1 Summary of the studies included in the database.

Table S2 All species and their trait values (number of observations, average, minimum and maximum).
Table S3 Summary results for all pairwise trait-trait relationships within and between species.

Please note: Wiley Blackwell are not responsible for the content or functionality of any Supporting Information supplied by the authors. Any queries (other than missing material) should be directed to the New Phytologist Central Office.

\section{About New Phytologist}

- New Phytologist is an electronic (online-only) journal owned by the New Phytologist Foundation, a not-for-profit organization dedicated to the promotion of plant science, facilitating projects from symposia to free access for our Tansley reviews and Tansley insights.

- Regular papers, Letters, Viewpoints, Research reviews, Rapid reports and both Modelling/Theory and Methods papers are encouraged. We are committed to rapid processing, from online submission through to publication 'as ready' via Early View our average time to decision is $<26$ days. There are no page or colour charges and a PDF version will be provided for each article.

- The journal is available online at Wiley Online Library. Visit www.newphytologist.com to search the articles and register for table of contents email alerts.

- If you have any questions, do get in touch with Central Office (np-centraloffice@lancaster.ac.uk) or, if it is more convenient, our USA Office (np-usaoffice@lancaster.ac.uk)

- For submission instructions, subscription and all the latest information visit www.newphytologist.com 